\title{
The Personal Mobility and Manipulation Appliance (PerMMA): a Robotic Wheelchair with Advanced Mobility and Manipulation
}

\author{
Hongwu Wang, Garrett G Grindle, Jorge Candiotti, Chengshiu Chung, Motoki Shino, Elaine Houston, \\ and Rory A Cooper, Fellow, IEEE
}

\begin{abstract}
The Personal Mobility and Manipulation Appliance (PerMMA) is a recently developed personal assistance robot developed to provide people with disabilities and older adults enhanced assistance in both mobility and manipulation, which are two fundamental components for independently activities of daily life performing, community participation, and quality of life. Technologies to assist with mobility and manipulation are among the most important tools for clinicians, end users and caregivers; however, there are currently few systems that provide practical and coordinated assistance with mobility and manipulation tasks. The PerMMA was not only developed and evaluated to provide users and caregivers enhanced mobility and manipulation options, but also as a clinical tool as well as research platform. The development and evaluation of PerMMA are presented in the paper.
\end{abstract}

\section{INTRODUCTION}

For many people with disabilities, there are architectural barriers existing in many cities and buildings during daily life, and it is expensive and time consuming to eliminate all of them. Thus, there are physical environmental barriers preventing electrical powered wheelchair (EPW) users from visiting friends and family [1] and carrying out daily activities [2, 3] - especially with regards to stairs, doorsteps, etc. In addition, essential tasks such as dressing, preparing food, shopping, or taking medications may require the assistance of a caregiver. For those who have concomitant mobility impairments, the use of a device that provides independent mobility and assistance with personal tasks could have a large impact on activity, participation, and quality of life and could reduce reliance on caregivers [4]. Technology which aids in these tasks must allow the user to independently control mobility, manipulation, and work within an unstructured environment [5]. The need for such integrated assistive technology systems is steadily growing. In the 2005 U.S. Census, over 11 million people reported a problem reaching overhead, and an equal number needed

Research supported by the National Science Foundation, the US Department of Veterans Affairs and the National Institute for Disability and Rehabilitation Research

Hongwu Wang, Garrett G Grindle, Jorge Candiotti, Chengshiu Chung, Elaine Huston, Dan Ding, are with the Human Engineering Research Laboratories, VA Pittsburgh Healthcare System and University of Pittsburgh, Pittsburgh, PA 15206 USA.

Motoki Shino is with the Department of Mechanical Engineering, the University of Tokyo, Tokyo, Japan.

Rory A Cooper. Author is with the Human Engineering Research Laboratories, VA Pittsburgh Healthcare System and University of Pittsburgh, Pittsburgh, PA 15206 USA (phone: 412-822-3700; fax: 412822-3699; e-mail: rcooper@pitt.edu). assistance with activities of daily living (ADLs) or instrumental activities of daily living (IADLs) [6].

Robotic systems have emerged as a rehabilitation engineering solution to ameliorate disabling conditions. Examples of such systems are the Deskto Vocational Assistant Robot, the Professional Vocational Assistant Robot, the "My Spoon", the Care-O-bot 3, the PR-2, the Manus ARM, the DORA and the JACO for enhancing manipulation assistance [7] and the TopChair, the Explorer, and the iBOT for increasing mobility support to assist wheelchair user climbing stairs [8]. However, few systems have been developed and evaluated with integrated mobility and manipulation support.

PerMMA is a new personal assistive robot, composed of a robotic powered wheelchair [9] and two Manus ARMs to provide enhanced mobility and bimanual manipulation for people with lower and upper extremity impairment (LUEI) [10]. The first generation of PerMMA (Gen I PerMMA) was developed with focus on proving user and caregivers with enhanced manipulation. The second generation of PerMMA (Gen II PerMMA) was developed for advance mobility control such as traction control and stair climbing. In this paper, the development and evaluation of both Gen I and Gen II PerMMA are presented in detail.

\section{DEVELOPMENT OF PERMMA}

\section{A. Development of Gen I PerMMA}

Gen I PerMMA (see Figure 1) includes:

a. Mobility. Gen I PerMMA uses a PerMobil ${ }^{\circledR} \quad$ C500 EPW (PerMobil Inc., USA) as its mobile base so that its robustness and reliability is assured. The C500 EPW provides a maximal payload capacity of $120 \mathrm{~kg}$. It includes four powered seating functions, seat back recline, seat tilt, elevating legrests, and seat elevation. All original equipped manufacturer (OEM)

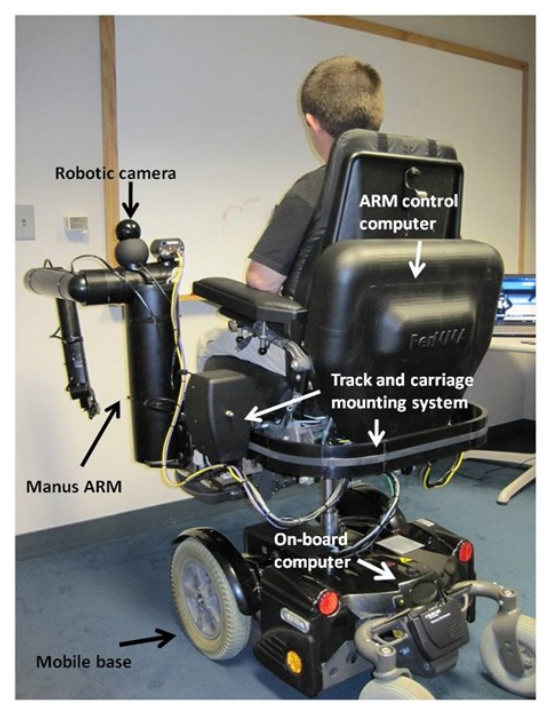

Figure 1 Gen I PerMMA overview 
electronics were removed and replaced with customized devices for integrating advanced features, such as manipulation and sensing.

b. Manipulation. Two Manus Assistive Robot Manipulators (Exact Dynamics Inc., Netherlands) were selected for PerMMA's manipulation capabilities. Each Manus ARM has a 6 degree of freedom (DoF) arm and a two-fingered gripper, and has a maximal reach radius of $830 \mathrm{~mm}$ around its shoulder and a vertical reach range from $385 \mathrm{~mm}$ to $1275 \mathrm{~mm}$ with respect to its base. Each ARM is capable of lifting a payload up to $2.5 \mathrm{~kg}$ when the arm is not outstretched. The ARM's previous FDA approval could help to secure PerMMA's FDA approval much faster and easier in its future commercialization.

c. Sensing. A variety of sensors are integrated to provide real-time feedback during its operation in order to enhance system intelligence as well as safety. A 6 DoF inertia sensor is incorporated into the seat base to detect vibration, roll rates, and wheel slip during driving. A web camera with tilt and pan control is mounted on the shoulder of each Manus ARM to provide vision feedback for the remote operator. $\mathrm{Wi}-\mathrm{Fi}$ and $3 \mathrm{G}$ accessibility are integrated to allow the system

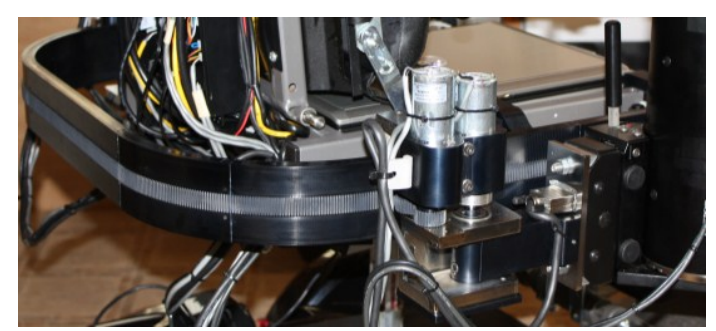

Figure 2 Track and carriage system for interfacing the robotic arms to the base

to communicate with computers and sensors.

d. Integration. In order to integrate the ARMs with the mobile base, a custom track and carriage system was designed and developed, as shown in Figure 2. Different from classic mounting methods, this novel track and carriage system allows the two arms to move anywhere along three sides of the mobile base, and greatly increases the workspace of the system [10]. The sensors are instrumented without interfering with the use of both arms and the base.

e. User Interfaces. Two different user interfaces were developed. The OEM joystick of the C500 EPW and two EPW seating function control boxes were adapted as the local control interface for mobility and seating functions. A touchpad (IntelliTools, USA, Figure 3) was chosen to be the control interface for the two arms. A remote operation station (Figure 3) was developed to allow a family member or a caregiver to remotely operate Gen I PerMMA. The remote operator uses an OEM computer keyboard to remotely control the driving speed and direction of the base, and uses two Phantom Omni haptic joysticks (SensAble Technologies Inc., USA) to control movements of both Manus ARMs. The similar physical structure of the haptic joystick and the Manus ARM makes manipulation more intuitive, easy to learn, and predictable for the remote operator. Each haptic joystick provides 6 DoFs, and each of them is mapped to control one DoF of the Manus ARM.

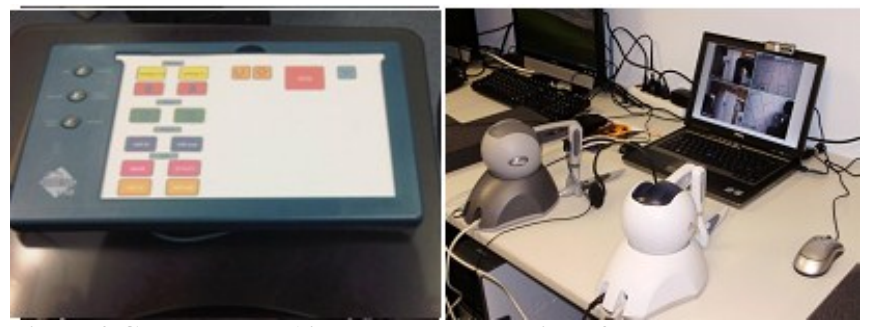

Figure 3 Gen I PerMMA's local and remote interface

f. Control Mode. Multiple control modes have been designed: local user (LU) mode, remote user (RU) mode, and cooperative control mode. In the LU mode, the local user has full control of PerMMA. The user can drive PerMMA using the wheelchair joystick, adjust the seat using all four seating functions by control box, relocate both ARMs along the track using a track control box, and operate both ARMs with the touchpad. In RU mode, a remote operator, who could be a caregiver of the local user, has full control of PerMMA's mobility and manipulation. This allows caregivers to remotely complete ADL for the local user. In cooperative mode, a local user and a remote operator work together to complete mobility and manipulation tasks. The local user first decides which function that he or she wishes to delegate to the remote assistant.

g. Safety Features. Gen I PerMMA has three levels of features aimed at mitigating risk of injury to the occupant. First, the software level includes soft stops by pushing a button on keyboard and keypad; keep-out zones to protect the local user from colliding with the arms; and a limited maximum speed of the mobile base and movement of arms. Second, the mechanical and electronic level includes a clean and neat design; sharp edges, hidden wires and moving parts have also been removed or covered. Third, the system level includes an independent cut-off switch available for the local user to shut off power to the whole system.

\section{B. Development of Gen II PerMMA}

Gen II PerMMA is designed with reconfigurable drive and caster wheels, which allows it to retain a small footprint for indoor use as well as traverse many difficult terrains (e.g., curbs, stairs, and gravel) with enhanced traction (Figure 4).

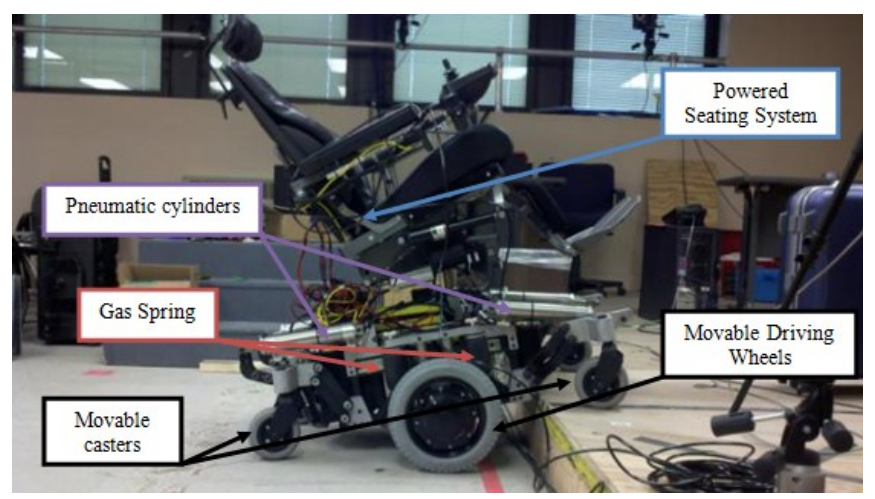

Figure 4 Gen II PerMMA overview 


\begin{tabular}{|c|c|c|c|c|c|}
\hline & $\begin{array}{l}\text { T1 - Retrieve a piece of } \\
\text { tissue from a tissue box } \\
\text { on a desk. (seconds) }\end{array}$ & $\begin{array}{l}\text { T2 - Pick up a meal container } \\
\text { and from a surface and move } \\
\text { to a defined location(seconds) }\end{array}$ & $\begin{array}{l}\text { T3 - Open a microwave } \\
\text { oven by pushing the door } \\
\text { button. (seconds) }\end{array}$ & $\begin{array}{l}\text { T4 - Retrieve a plastic cup } \\
\text { and move it close enough for } \\
\text { the user to drink. (seconds) }\end{array}$ & $\begin{array}{l}\text { T5 - Retrieve a straw } \\
\text { and put it into a } \\
\text { plastic cup. (seconds) }\end{array}$ \\
\hline Local & $350 \pm 195$ & $297 \pm 157$ & $192 \pm 151$ & $275 \pm 44$ & $418 \pm 307$ \\
\hline Coop & $136.4 \pm 60.13$ & $124 \pm 49$ & $70 \pm 31$ & $70 \pm 12$ & $220 \pm 140$ \\
\hline
\end{tabular}

The Gen II PerMMA mobile base uses a six-wheel design, similar to many current electric powered wheelchairs. The front and rear wheel casters are mounted to the main frame via 4-bar linkages. The position of the front and rear casters is controlled via four independent pneumatic actuators. The drive wheels use hub-motors and are mounted to the frame via a sliding platform, which allows the drive wheels to be moved up/down independently with pneumatic actuators, and fore-aft to alter the center of mass and driving dynamics with a carriage and an electric linear actuator.

The Gen II PerMMA electrical design was expanded with more electrical components from Gen I: a relay board to control the seating functions; a relay board to control the pneumatic cylinders for moving the four casters and driving wheel up and down; the electrical motor for moving the driving wheel forward and backward; a replay board to control the caster brakes during curb and stair climbing; a PWM generation board to generate a PWM signal to control the pneumatic system; a circuit board to control the caster brakes; and a digital I/O board for receiving feedback from the pneumatic cylinders. All the pneumatics, electrical motors, caster brakes, and power seating functions can be controlled either by the on-board single board computer through digital I/Os or by a keypad through relay boards.

The Gen II PerMMA is controlled in two modes: Advanced Driving Mode (ADM) and Automatic Climbing Mode (ACM) In ADM, when the wheelchair moves on flat or uneven terrain, the two motorized driving wheels are always in contact with the terrain. The caster wheels can be configured for front-wheel drive (rear caster wheels in contact with the terrain), rear-wheel drive (front caster wheels in contact with the terrain), or middle-wheel drive (both front and rear caster wheels in contact with terrain). While the wheelchair would be stable with all the six wheels in contact with the ground (especially during off road travel), this configuration is not optimized for traversing obstacles such as curbs, grass, gravel, uneven terrain and snow compared with front-wheel drive configuration. In ACM during stair climbing operations, more importance is given to wheelchair stability and so all the four caster wheels are in contact with the ground when the driving wheels are lifting up for stair climbing (as shown in Fig. 5). Caster brakes are engaged when the driving wheels are not in contact with the ground by using pressure sensors. A mathematical model of Gen II PerMMA was developed and a feedback and feedforward controller for stability of the system was designed (Figure 6).

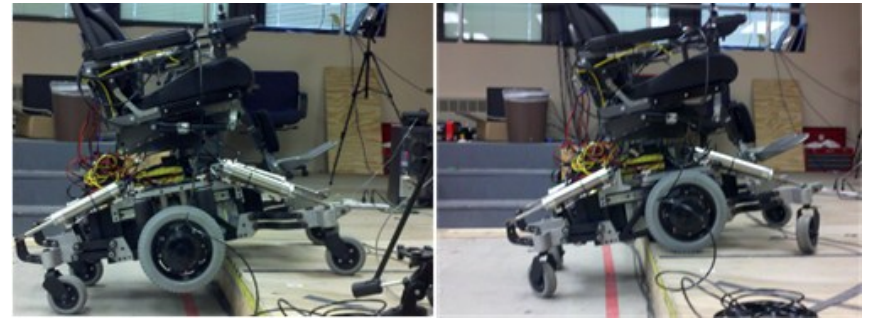

Figure 5 Gen II PerMMA in automatic climbing mode

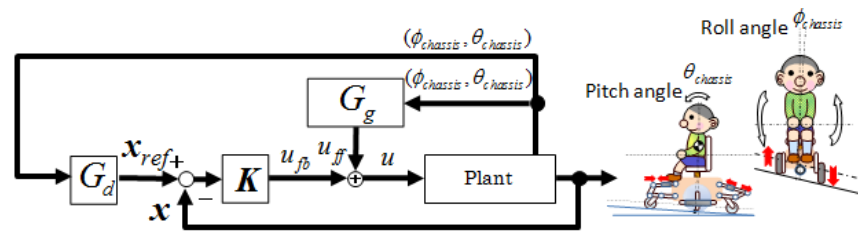

Figure 6 Feedback and feed-forward control diagram for Gen II PerMMA

\section{Evaluation OF PERMMA}

Fifteen participants ( 9 male and 6 female) were recruited to evaluate the basic operation of Gen I PerMMA within a laboratory environment. The study was approved by the University of Pittsburgh Institutional Review Board. The age of the participants was $42.9 \pm 15.8$ (means \pm standard deviation through all the paper) years. All participants had both upper and lower extremity impairments and were electric powered wheelchair users.

Participants were asked to complete five tasks independently using both local and cooperative control modes after 1 hour training). The mean time to complete the five tasks alone and in cooperation with a remote operator is provided in Table 1. Despite the results showing that cooperative control was much faster, when surveyed, nearly all participants preferred operating PerMMA independently.

A working prototype of Gen II PerMMA has been developed with 90.7 Kilogram weight, 0.36 meter driving wheel diameter and up to 2.7 meter/second velocity. The overall dimensions of Gen II PerMMA (Gen I is using the commercial available EPW as a base so it is not included here) are comparable to market available EPWs (Table 2).

Table 2 Gen II PerMMA dimension compare with other EPWs

\begin{tabular}{|c|c|c|}
\hline Wheelchair Model & Length (meters) & Width (meters) \\
\hline Gen II PerMMA & 1.02 & 0.66 \\
\hline Permobil C500 & 1.26 & 0.65 \\
\hline Invacare TDX & 0.90 & 0.65 \\
\hline RESNA dimensions & 1.20 & 0.70 \\
\hline
\end{tabular}


Simulations of Gen II PerMMA climbing an 8 " curb and driving over an obstacle were conducted using the Open Dynamic Engine (ODE) software package. The simulations identified a force based control scheme as the most effective for climbing curbs/steps (Figure 7). Pressure sensors in each of the pneumatic actuators can be used to measure surface contact and to sequence the control algorithm. The stability control simulation results showed that with the controller applied, there was less pitch and roll motion than by controlling the vertical driving wheel position and the caster arms simultaneously without the control (Figure 8). The dark line represents the result with the control applied and the light line represents angles without control. There was less deviation for the pitch angle with the control applied than without the control. When the wheelchair was driven on a slope, the roll angle was within 3 degrees with the control applied, while without the control the roll angle was more than 8 degrees.

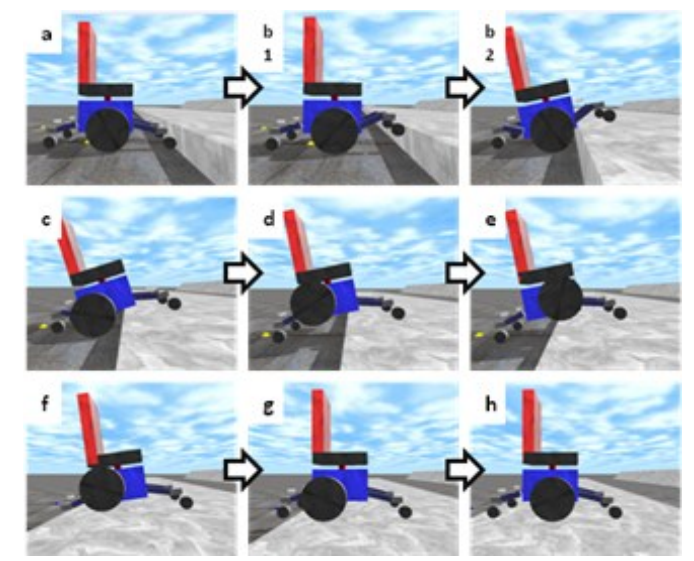

Figure 7 Gen II PerMMA climbing 8" curb

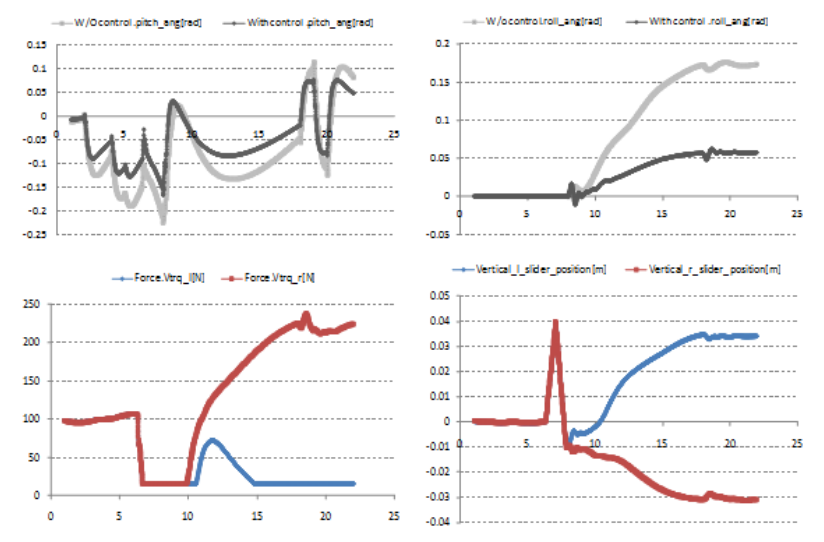

Figure 8 Simulation results of Gen II PerMMA stability control

\section{CONCLUSIONS}

Gen I PerMMA has been evaluated by end users, care givers, and clinicians. With the two robotic arms and diverse control interface, the user could conduct more activities of daily life and reported higher satisfaction. The preliminary study only had 15 subjects but more clinical testing has been proposed and approved by IRB for Gen I PerMMA user interface and remote operation. In addition, with the expandability of our design, Gen I PerMMA is not only used as a clinical tool, but also as a research platform.

For Gen II PerMMA, experimental testing is in progress to validate the design and stability control. In addition, simulation of Gen II PerMMA climbing up to four steps is undergoing. After additional testing and safety checking, clinical trials will be conducted to evaluate the usability of Gen II PerMMA.

At the same time, our ultimate goal is to integrate Gen I and Gen II PerMMA manipulation and mobility together into the Gen III PerMMA which provides both advance mobility and manipulation.

\section{ACKNOWLEDGMENTS}

The authors would like to thank Zachary Merrill, Josh Brown, Annmarie Kelleher and Rosemarie Cooper for their valuable help on this work. This material is based upon work supported in part by the National Science Foundation Grant\#EEC-540865, Office of Research and Development, Rehabilitation Research \& Development Service, Department of Veterans Affairs, Grant\#B3142C and \#B6789C, and National Institute for Disability and Rehabilitation Research Grant\#H133P09001002. The contents of this paper do not represent the views of the Department of Veterans Affairs or the United States Government.

\section{REFERENCES}

[1] A. Brandt, S. Iwarsson, and A. Stahle. "Older people's use of powered wheelchairs for activity and participation," J Rehabil Med. vol. 36, no.2, pp.70-77, 2004 Mar

[2] D. Field. "Powered mobility: a literature review illustrating the importance of a multifaceted approach," Asst Technol, vol.11, no.1, pp.20-33, 1999.

[3] M.E. Buning, J.A. Angelo, M.R. Schmeler. "Occupational performance and the transition to powered mobility: a pilot study," Am J Occup Ther, vol.55, no.3, pp.339-344, 2001May

[4] W. Erickson, C. Lee, S. von Schrader. "2009 Disability Status Report: United States," Ithaca, NY: Cornell University Employment and Disability Institute (EDI), 2011

[5] R.I. Stone, J.M. Wiener. "Who Will Care for Us? Addressing the Long-term Care Workforce Crisis. Urban Institute," Available from : http://www.urban.org/publications/310304.html, 2010

[6] J.M. McNeil. "Americans with disabilities: 2005, household economic studies, current population reports," pp. 70-117.2008

[7] R.A. Cooper, G.G. Grindle, J.J. Vazquez, J. Xu, H. Wang, J. Candiotti, B.A. Salatin, E. Houston, A.R. Kelleher, R.M. Cooper, E. Teodorski, S. Beach, "Personal Mobility and Manipulation Appliance (PerMMA) - Design, Development and Initial Testing," Proceedings of the IEEE, in press

[8] H. Wang, J. Xu, G.G. Grindle, J.J. Vazquez, B. Salatin, D. Ding, R.A. Cooper. "Performance evaluation of the personal mobility and manipulation appliance," Med Eng Phys, submitted for publication

[9] H. Wang, B. Salatin, G.G. Grindle, D. Ding, R.A. Cooper. "RealTime Model-Based Electric Powered Wheelchair Control," Med Eng Phys, vol. 31, no.10, pp.1244-1254, 2009 Sep.

[10] G.G. Grindle, H. Wang, B. Salatin, J.J.Vazquez, R.A. Cooper, "Design and Development of the Personal Mobility and Manipulation Appliance (PerMMA)," Assistive Technology, Vol. 23, No. 2, pp. 81-92, June 2011. 